\title{
Vaccination with a Recombinant Fragment of Collagen Adhesin Provides Protection against Staphylococcus Aureus-mediated Septic Death
}

\author{
Ing-Marie Nilsson, ${ }^{\star}$ Joseph M. Patti, ${ }^{\ddagger}$ Tomas Bremell, ${ }^{*}$ Magnus Höök, ${ }^{\ddagger}$ and Andrzej Tarkowski ${ }^{\star}$ \\ $*$ Department of Rheumatology, University of Göteborg, S-41346 Sweden; ${ }^{\ddagger}$ Center for Extracellular Matrix Biology, Institute of Biosciences \\ and Technology, Texas A\&M University, Houston, Texas 77030
}

\begin{abstract}
Staphylococcus aureus is a major cause of nosocomial and community-acquired infections. Morbidity and mortality due to infections such as sepsis, osteomyelitis, septic arthritis, and invasive endocarditis remain high despite the use of antibiotics. The emergence of antibiotic resistant super bugs mandates that alternative strategies for the prevention and treatment of $S$. aureus infections are developed. We investigated the ability of vaccination with a recombinant fragment of the $S$. aureus collagen adhesin to protect mice against sepsis-induced death. Actively immunized NMRI mice were intravenously inoculated with the $S$. aureus clinical isolate strain Phillips. $14 \mathrm{~d}$ after inoculation, mortality in the collagen adhesin-vaccinated group was only $13 \%$, compared with $87 \%$ in the control antigen immunized group $(P<0.001)$. To determine if the protective effect was antibody mediated, we passively immunized naive mice with collagen adhesin-specific antibodies. Similar to the active immunization strategy, passive transfer of collagen adhesin-specific antibodies protected mice against sepsisinduced death. In vitro experiments indicated that $S$. $a u$ reus opsonized with sera from collagen adhesin immunized mice promoted phagocytic uptake and enhanced intracellular killing compared with bacteria opsonized with sera from control animals. These results indicate that the collagen adhesin is a viable target in the development of immunotherapeutics against $S$. aureus. (J. Clin. Invest. 1998. 101:26402649.) Key words: sepsis • antibody response • phagocytosis • intracellular killing • Staphylococcus aureus
\end{abstract}

\section{Introduction}

Staphylococcus aureus expresses a repertoire of factors including adhesins, exotoxins, exoenzymes, capsular polysaccharides, and numerous cell-associated components that contribute to the pathogenesis of disease (1). This elaborate array of virulence factors promotes the ability of $S$. aureus to cause disease in humans that range from a localized skin infection such as

Address correspondence to Ing-Marie Nilsson, Department of Rheumatology, Guldhedsgatan 10, S-41346 Göteborg, Sweden. Phone: 4631-604616; FAX: 46-31-826791; E-mail: Ing-Marie.Nilsson@immuno. gu.se Joseph M. Patti's current address is Inhibitex, Inc., 35 Technology Parkway South, Suite 170, Norcross, GA 30092.

Received for publication 25 September 1997 and accepted in revised form 15 April 1998.

J. Clin. Invest.

(C) The American Society for Clinical Investigation, Inc. 0021-9738/98/06/2640/10 \$2.00

Volume 101, Number 12, June 1998, 2640-2649

http://www.jci.org impetigo to systemic life threatening conditions that include osteomyelitis and infective endocarditis. The dramatic increase in the number of antibiotic multiresistant staphylococci coupled with the slow development of new anti-infective agents has renewed interest in the development of immunotherapeutics directed against $S$. aureus.

The initial critical event in most staphylococcal infections is the ability of bacteria to adhere to components within host tissues. The specific interaction between $S$. aureus and extracellular matrix components, such as fibrinogen, fibronectin, or collagen is mediated by specific microbial cell surface components recognizing adhesive matrix molecules $(2,3)$. Genes encoding the fibronectin (4), fibrinogen (5), elastin (6), and collagen (7) binding adhesins have been cloned, sequenced, and in most cases the corresponding recombinant proteins characterized. The biological role of $S$. aureus adhesin as important virulence factors contributing to the initiation of disease is now clearly established. Adhesion of $S$. aureus to indwelling catheters, orthopedic implants, and cardiovascular devices is seen as the critical step in the development of device-related infections (8). S. aureus adheres to the implants by attaching to the host plasma components and activated platelets that are rapidly deposited on the surface of the foreign body (9). Several in vitro studies have shown that $S$. aureus' adherence to polymeric surfaces is strongly promoted by plasma proteins such as fibronectin, fibrinogen, and vitronectin. The clinical relevance of these in vitro experiments has been documented by clinical studies $(10,11)$ showing the in vivo contribution of fibronectin, fibrin, and fibrinogen to $S$. aureus attachment to catheters inserted in hospitalized patients.

The construction of $S$. aureus collagen adhesin isogenic mutants and their use in animal models has also helped to delineate the roles that adhesins play in the initiation of infection. For example, the biological importance of a collagen adhesin in the pathogenesis of hematogenously spread infections has been examined by comparing the virulence of $S$. aureus cna mutants in an animal model of septic arthritis (12) and endocarditis (13). In both models, the collagen adhesin negative isogenic mutant was significantly less virulent when compared with the parental strain.

Because bacterial adherence is the first critical step in the development of most infections, it is an attractive target for the development of novel vaccines. To determine if a collagen adhesin-based vaccine could prevent against sepsis-induced death, mice were actively immunized with a recombinant fragment of the collagen adhesin and challenged intravenously with $S$. aureus. The protective effect of collagen adhesin specific antibodies was confirmed by passive immunization studies in mice.

\section{Methods}

Mice. Male NMRI mice, 5-8 wk old, were obtained from B\&K Universal AB (Sollentuna, Sweden) and maintained in the animal facility 
of the Department of Rheumatology, University of Göteborg, Sweden. They were housed ten to a cage under standard conditions of temperature and light, and fed standard laboratory chow and water ad libitum.

Bacterial strains. S. aureus strain Phillips $\left(\mathrm{CNA}^{+}\right.$; reference 12$)$, is a clinical osteomyelitis isolate that expresses the collagen adhesin. S. aureus LS-1 ( $\left.\mathrm{CNA}^{-}\right)$, is a strain that does not express a collagen adhesin and was originally isolated from a swollen joint of a spontaneously arthritic mouse (14). Before the animal experiments, $S$. aureus strains were cultured on blood agar plates for $24 \mathrm{~h}$, and then streaked for isolated colonies on a fresh blood agar plate and incubated for an additional $24 \mathrm{~h}$. The bacteria were harvested from the agar plate in PBS containing 5\% BSA and 10\% dimethyl sulfoxide and kept frozen at $-20^{\circ} \mathrm{C}$. Before inoculating animals, the bacterial solution was thawed, washed, diluted in PBS, and adjusted to the appropriate concentration. Viable counts were used to determine the exact number of bacteria inoculated into the mice.

Construction of expression plasmids. Collagen adhesin gene fragments from $S$. aureus strain FDA 574 were overexpressed in Escherichia coli using the vector pQE-30 (QIAGEN Inc., Chatsworth, CA). Recombinant proteins expressed from this vector contain an $\mathrm{NH}_{2}$-terminal tail of six histidine residues. The construction of expression constructs M17 (collagen binding domain [CBD $\left.]^{1}[151-297]\right)$, M31 (CBD[61-343]), and M55 (CBD[30-529]) have been described previously $(15,16)$.

Expression and purification of recombinant proteins. Overnight cultures of $E$. coli JM101 cells harboring the recombinant plasmids were diluted 1:50 in 1 liter of Luria Broth (GIBCO BRL, Gaithersburg, MD) containing $50 \mu \mathrm{g} / \mathrm{ml}$ ampicillin. E. coli cells were grown until the culture reached an $\mathrm{OD}_{600}$ of $0.5-0.8$. Expression of the recombinant proteins was then induced by adding IPTG to a final concentration of $0.2 \mathrm{mM}$. After a 3-h induction period, the bacteria were collected by centrifugation and the bacterial pellets were resuspended in buffer A $(5 \mathrm{mM}$ imidazole, $0.5 \mathrm{M} \mathrm{NaCl}, 20 \mathrm{mM}$ Tris, $\mathrm{pH}$ 7.9). The cells were lysed by passage through a French press twice at $20,000 \mathrm{lb} / \mathrm{in}^{2}$. The bacterial lysates were centrifuged at $102,000 \mathrm{~g}$ for 10 min to remove bacterial debris. The supernatants containing soluble proteins was filtered through a $0.45 \mu \mathrm{M}$ membrane (Corning Glass Works; Corning, NY) and retained for further purification. The recombinant proteins were purified by immobilized metal chelate affinity chromatography. A column containing iminodiacetic acid Sepharose $6 \mathrm{~B}^{\circledR}$ Fast Flow (Sigma Chemical Co., St. Louis, MO), connected to a FPLC system (Pharmacia LKB Biotechnology, Inc., Piscataway, $\mathrm{NJ}$ ), was charged with $150 \mathrm{mM} \mathrm{Ni}^{+2}$ and equilibrated with buffer A. After equilibration, the bacterial supernatant was applied to the column and the column was washed with 10 bed volumes of buffer A. Subsequently, the column was eluted with buffer B (200 mM imidazole, $0.5 \mathrm{M} \mathrm{NaCl}, 20 \mathrm{mM}$ Tris, $\mathrm{pH}$ 7.9). The eluate was monitored for protein by the absorbance at $280 \mathrm{~nm}$ and peak fractions were analyzed by SDS-PAGE.

Immunization of mice and experimental protocol-active immunization. Purified recombinant collagen adhesin fragments M17, M31, M55, and BSA (Sigma Chemical Co.) were dissolved in PBS and emulsified 1:1 in Freund's complete adjuvant (Difco Laboratories, Inc., Detroit, MI). $200 \mu \mathrm{l}$ of the emulsion containing $100 \mu \mathrm{g}$ of protein was injected subcutaneously (s.c.) on day -31 . Booster immunizations (s.c.; $100 \mu \mathrm{g}$ protein in PBS) were performed 15 and $24 \mathrm{~d}$ after the initial vaccination. After the final boost, mice were inoculated intravenously (i.v.) with S. aureus. The mice were weighed and blood samples were obtained before bacterial challenge. Four separate vaccination experiments were conducted. In the first experiment, the mice were immunized with M17 $(n=15), \operatorname{M} 31(n=14), \operatorname{M} 55(n=$ $11)$, or BSA $(n=15)$, and inoculated with $2.8 \times 10^{7} \mathrm{CFU}$ per mouse of S. aureus strain Phillips. In the second and third experiments, mice were immunized with M55 or BSA and then challenged with $2 \times 10^{7}$

1. Abbreviation used in this paper: $\mathrm{CBD}$, collagen binding domain. or $6 \times 10^{6} \mathrm{CFU}$ per mouse S. aureus strain Phillips, respectively. Blood samples and bacterial cultures from kidneys and a standard pair of joints (right ankle and wrist) were obtained at the day the mice were killed. In the fourth experiment, the mice were immunized with M55 or BSA and then inoculated with $2.8 \times 10^{7} \mathrm{CFU}$ per mouse S. aureus strain LS-1.

Passive immunization. To generate anticollagen adhesin antibodies, 10 rats were immunized with M55 as described above. For generation of the control antibodies, eight rats were immunized with BSA. All the rats were bled by heart puncture, sera were pooled, and the IgG fraction was obtained by precipitation with a saturated ammonium sulphate solution, followed by extensive dialysis against PBS. The concentration of immunoglobulins in the precipitate was determined by single radial immunodiffusion technique (17). Naive mice were passively immunized i.p. on day -1 with $16 \mathrm{mg}$ of the immunoglobulin fraction containing antibodies specific for either M55 $(n=$ $10)$ or BSA $(n=10)$. On day 0 , the mice were challenged i.v. with $4 \times$ $10^{7}$ CFU per mouse $S$. aureus strain Phillips and mortality was monitored over the next $7 \mathrm{~d}$.

Bacteriological examination of infected animals. Mice were immunized with M55 $(n=16)$ or BSA $(n=15)$ and subsequently infected with $6 \times 10^{6} \mathrm{CFU}$ per mouse. $14 \mathrm{~d}$ after bacterial challenge, the mice were killed and one standard pair of joints (right ankle and wrist) was dissected aseptically and bacterial samples were obtained with charcoaled sticks and then streaked on blood agar plates and incubated for $48 \mathrm{~h}$ at $37^{\circ} \mathrm{C}$. To avoid false-positive results due to contamination, a joint was considered infected only when more than 20 S. aureus colonies were recovered (18). The kidneys were aseptically removed, homogenized, and diluted to appropriate volume in PBS. $100 \mu \mathrm{l}$ of the homogenate was transferred to agar plates and the plates were incubated for $24 \mathrm{~h}$ and the number of CFU were determined. Bacteria were also tested for catalase and coagulase activity.

Serological analyses IL-6 levels. The murine hybridoma cell line B9, which is dependent on IL-6 for its growth, was used to determine the serum levels of IL-6 $(19,20)$. The B9 cells were seeded into microtiter plates $(5,000$ cells per well) and dilutions of the serum samples were added to the wells. After a 68 -h incubation, $\left[{ }^{3} \mathrm{H}\right]$ thymidine $(\mathrm{Ra}-$ diochemical Centre; Amersham International, Little Chalfont, UK) was added and $6 \mathrm{~h}$ later the cells were harvested. The results were compared with an IL-6 standard. Previously, the B9 cells were shown not to react with several recombinant cytokines including IL- $1 \alpha$, IL$1 \beta$, IL-2, IL-3, IL-5, granulocyte/macrophage-CSF, TNF- $\alpha$, and IFN- $\gamma$. There was only a weak reactivity with IL-4 (20).

Specific antibodies. The serum level of specific antibodies against the collagen adhesin fragment M55 and M31 was measured by an ELISA. Sera from hyperimmunized mice were obtained 1 wk after the last booster immunization, whereas sera from infected mice were obtained $3 \mathrm{wk}$ after the bacterial inoculation. 96-well microplates (Nunc A/S, Roskilde, Denmark) were coated overnight at $4^{\circ} \mathrm{C}$ with M55 protein $(2 \mu \mathrm{g} / \mathrm{ml})$ in PBS. The plates were blocked with $0.5 \%$ ovalbumin (Sigma Chemical Co.) dissolved in 0.05 M Tris ( $\mathrm{pH} 7.7$ ). Sera, biotinylated antibodies, and ExtrAvidin-peroxidase (Sigma Chemical Co.) were all diluted in 0.05 M Tris ( $\mathrm{pH} 7.4), 0.015 \mathrm{M} \mathrm{NaCl}$. The plates were incubated overnight at $4^{\circ} \mathrm{C}$ with sera, washed and incubated stepwise with biotinylated goat anti-mouse IgG antibody (Jackson ImmunoResearch Laboratories, Inc., West Grove, PA), ExtrAvidin-peroxidase $(0.5 \mu \mathrm{g} / \mathrm{ml}$; Sigma Chemical Co. $)$ and ABTS substrate. The $\mathrm{A}_{405}$ was measured in a Titertec Multiscan photometer (Flow Laboratories, McLean, VA). A similar ELISA procedure as described above was employed to detect antibodies to M17, M31, as well as to the B1 domain of collagen adhesin. Ovalbumin was used as a control antigen. In addition to the antigen-based ELISA, 96-well plates were precoated with poly-L-lysine (Sigma Chemical Co.) and then coated with either $1.5 \times 10^{7}$ of formalin killed $S$. aureus strains Phillips or LS-1. After blocking the unreacted sites with ovalbumin, serum-pooled from M55 immunized mice, unimmunized mice, and mice unimmunized but experimentally infected with $S$. aureus strain Phillips, respectively was added to the immobilized bacteria. The de- 
velopment steps were completed as described above. Irrespective of the coating specificity, the sera were assayed at several dilutions. The highest dilution of serum representing an absorbance found within the linear part of the standard curve is presented in Fig. 4. In the case of anti-S. aureus antibodies, the serum dilution was 1:20,000, whereas the corresponding serum dilution in case of recombinant staphylococcal antigens and OVA was either 1:20,000 (see Fig. $5 A$ ) or 1:5,000 (Fig. $5 B$ ), respectively.

Opsonization, phagocytosis, and intracellular killing of bacteria. The phagocytosis assay was done by a modification of the method described previously (21). Briefly, macrophages originating from naive mice were collected from the peritoneal cavity by injecting $3 \mathrm{ml}$ of ice-cold medium (Iscove's medium containing $10 \%$ FCS and $100 \mu \mathrm{g} / \mathrm{ml}$ of gentamicin). After a 1-min massage of the abdomen, the macrophages were aspirated with a syringe. The peritoneal macrophages were washed and adjusted to $2 \times 10^{6}$ cells per milliliter, seeded $(200 \mu \mathrm{l})$ into 24-well plates (Nunc A/S, Roskilde, Denmark) and left at room temperature for $90 \mathrm{~min}$. $500 \mu \mathrm{l}$ of cell culture medium was added to each well and the cells were incubated for $24 \mathrm{~h}$ in $37^{\circ} \mathrm{C}$. The medium was then removed and replaced by $500 \mu \mathrm{l}$ of a new medium free of antibiotics and the cells were then incubated overnight at $37^{\circ} \mathrm{C}$. The next day, S. aureus strain Phillips was opsonized for $30 \mathrm{~min}$ at $4^{\circ} \mathrm{C}$ with either heat inactivated sera from $(a)$ mice that have been hyperimmunized with M55; $(b)$ sera from BSA hyperimmunized mice or alternatively; or $(c)$ sera from unimmunized mice but experimentally infected with $S$. aureus strain Phillips. $500 \mu l$ of sera containing the opsonized staphylococci $\left(1.4 \times 10^{3}\right.$ bacteria per milliliter $)$ were added to the macrophages. After $50 \mathrm{~min}$ of incubation, the macrophages were washed three times in Iscove's medium in order to remove noningested bacteria. To determine the uptake of the opsonized $S$. aureus, macrophages were immediately analyzed. It should be noted that at this stage of experiment one cannot differentiate between adherent and ingested bacteria. To assess intracelullar killing of bacteria, another set of cultures was incubated for an additional $4 \mathrm{~h}$, in Iscove's medium containing minimal inhibitory concentration of gentamicin to avoid extracellular replication of bacteria. The macrophages were lysed with distilled water for $20 \mathrm{~min}$, and the lysate diluted $1: 1,1: 10,1: 100$, and 1:1,000 in PBS and cultured on 5\% blood agar plates. The plates were incubated overnight and the number of bacteria counted.

Statistical analysis. Statistical evaluation was done by using the Mann-Whitney U test or the chi-square test. Correlations between absorbance and weight decrease/mortality rates were assessed by Spearman's rank correlation test. Results are reported as means \pm SEMs.

\section{Results}

Clinical course of disease-active immunization. Previously, we have described the cloning and sequencing of the gene (cna) that encodes a $133-\mathrm{kD}$ S. aureus collagen adhesin (7). The collagen adhesin contains a signal sequence that is followed by a large region of unique sequence, denoted domain A. Immediately following the domain $\mathrm{A}$ is a 187 amino acid motif repeated three times. The carboxyl-terminal portion of the collagen adhesin consists of a 64-amino acid lysine and proline rich region, and a stretch of hydrophobic residues that may represent the membrane-spanning region followed by a cluster of positively charged amino acids. The consensus sequence LPXTG (22), found in most cell surface anchored proteins from Gram-positive bacteria, is present in the collagen adhesin. The ligand-binding domain of the $S$. aureus collagen adhesin was initially mapped to the $55-\mathrm{kD}$ A region. Molecular characterization of the cna gene has shown the 1.6-kb DNA segment encoding the A domain to be conserved among clinical $S$. aureus isolates that bind collagen, whereas the carboxyl half of the collagen adhesin can contain either one, two, three,

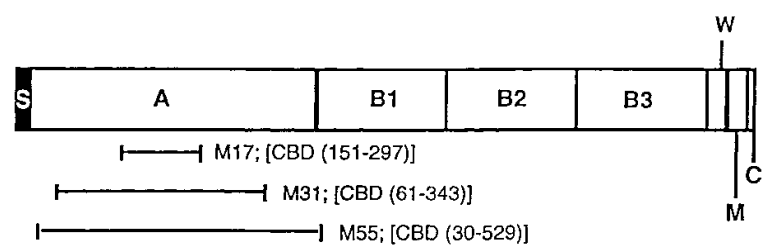

Figure 1. Model of the S. aureus collagen binding adhesin from S. aureus FDA 574 (7) and denotation of the recombinant versions of the collagen adhesin constructs used to immunize the animals. The amino acid residues contained within each construct are indicated.

or four B repeats of 167 amino acids (23). Using deletion mutagenesis in combination with Western ligand blots and direct binding assays, the collagen CBD was localized to a 168 amino acid long segment (CBD 151-318) within the $\mathrm{NH}_{2}$-terminal portion of the adhesin (16). A series of expression constructs derived from domain A of the collagen adhesin of $S$. aureus FDA 574 were tested for their ability to generate an immune response that would protect mice against a heterologous challenge with a lethal dose of $S$. aureus (Fig. 1). Mice were vaccinated subcutaneously three times with M17, M31, M55, or BSA. 1 wk after i.v. challenge with $2.8 \times 10^{7} \mathrm{CFU}$ per mouse S. aureus strain Phillips, $73 \%$ of the mice immunized with BSA had died, conversely only $27 \%$ of the mice vaccinated with M55 had died. An intermediate mortality rate of 53 and $50 \%$ was seen in the group of mice immunized with M17 or M31, respectively. $10 \mathrm{~d}$ after the i.v. bacterial challenge, all of the mice immunized with BSA had died, however the mortality rate in the M55 immunized group only reached 36\% $(P<0.01)$. The experiment was terminated at day 14 with no further deaths occurring among the M55 immunized mice (Fig. $2 A$ ). It is clear from these data that vaccination with M55, a polypeptide representing the entire A domain of the collagen adhesin, provided superior protection compared with the smaller recombinant proteins M17 and M31. Although not statistically significant, the smaller constructs exhibited modest protection against the lethal $S$. aureus i.v. challenge compared with BSA.

To further examine the ability of the recombinant domain A from $S$. aureus FDA 574 collagen adhesin to protect mice against $S$. aureus infection, an additional 30 mice were immunized subcutaneously with either M55 or BSA. The mice were then challenged i.v. with $2 \times 10^{7} \mathrm{CFU}$ per mouse $S$. aureus strain Phillips. $14 \mathrm{~d}$ after the inoculation 2/15 mice (13\%) were dead in the M55 immunized group, compared with 13/15 mice $(87 \%)$ in the control group $(P<0.001$; Fig. $2 B)$. The mice were followed for an additional $21 \mathrm{~d}$, however there were no further deaths in either group. In addition to an increase in the mortality rate, the control mice exhibited a significant decrease in their body weight compared with M55 immunized mice $(31.3 \pm 1.0 \%$ versus $27.1 \pm 1.6 \% ; P<0.05)$.

In the third experiment, mice were immunized with either M55 $(n=16)$ or BSA $(n=15)$ and challenged i.v. with $6 \times 10^{6}$ CFU per mouse $S$. aureus strain Phillips. The reduced number of microorganisms in the challenge inoculum resulted in mortality of only $6 \%$ of the M55 immunized mice (1/16). Conversely, $33 \%(5 / 15)$ of BSA immunized mice died within the $14 \mathrm{~d}$ after $S$. aureus challenge. As in the previous two experiments, mice vaccinated with BSA showed significantly greater weight loss during the course of infection (Fig. 3). Taken together, 

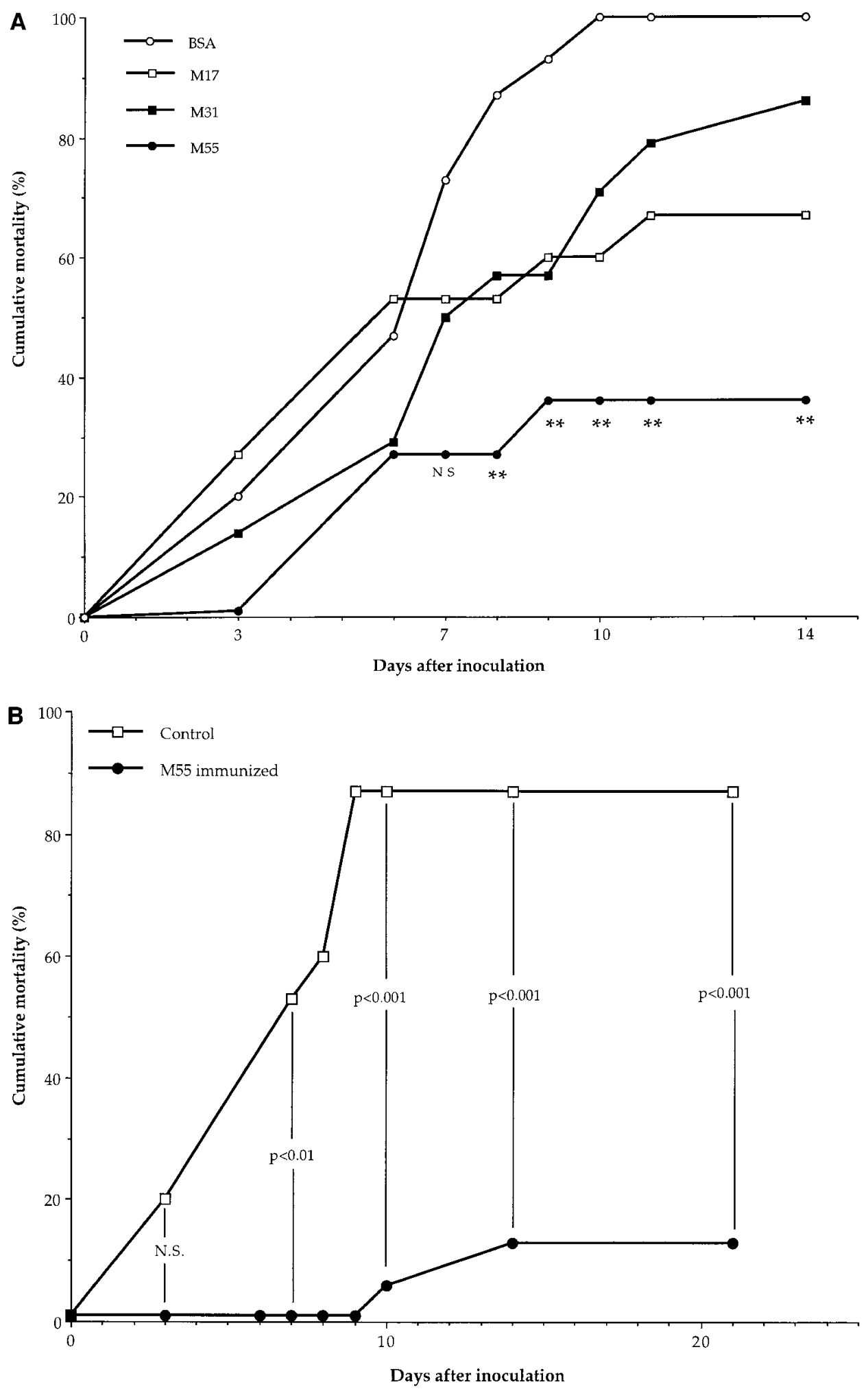

Figure 2. (A) Cumulative mortality in NMRI mice s.c. immunized with the recombinant collagen binding adhesin M55 $(n=11)$, M31 $(n=$ $15)$, M17 $(n=14)$, or BSA $(n=15)$ before i.v. challenge with $2.8 \times 10^{7}$ CFU per mouse $S$. aureus strain Phillips. $P$ values refer to comparisons between M55-immunized mice and BSA-immunized mice. $* * P<$ 0.01. (B) Cumulative mortality in NMRI mice after immunization with recombinant collagen binding adhesin M55 $(n=15)$ or BSA $(n=15)$ and subsequent i.v. challenge with $2 \times 10^{7} \mathrm{CFU}$ per mouse $S$. aureus strain Phillips.

these experiments clearly indicate that vaccination with the recombinant M55 fragment of collagen adhesin provides very efficient protection against a lethal heterologous $S$. aureus challenge. With respect to the development of septic arthritis, $3 \mathrm{~d}$ after bacterial inoculation $\left(6 \times 10^{6} \mathrm{~S}\right.$. aureus strain Phillips/ mouse) $8 / 15(53.3 \%)$ of BSA-immunized mice developed ar- thritis whereas the corresponding value for M55 immunized group was $6 / 16(37.5 \%)$. Similar values, although with overall lower frequency were obtained on day 14 after bacterial inoculation (data not shown).

To assess whether the protection seen in mice immunized with M55 was specifically related to an immune response tar- 


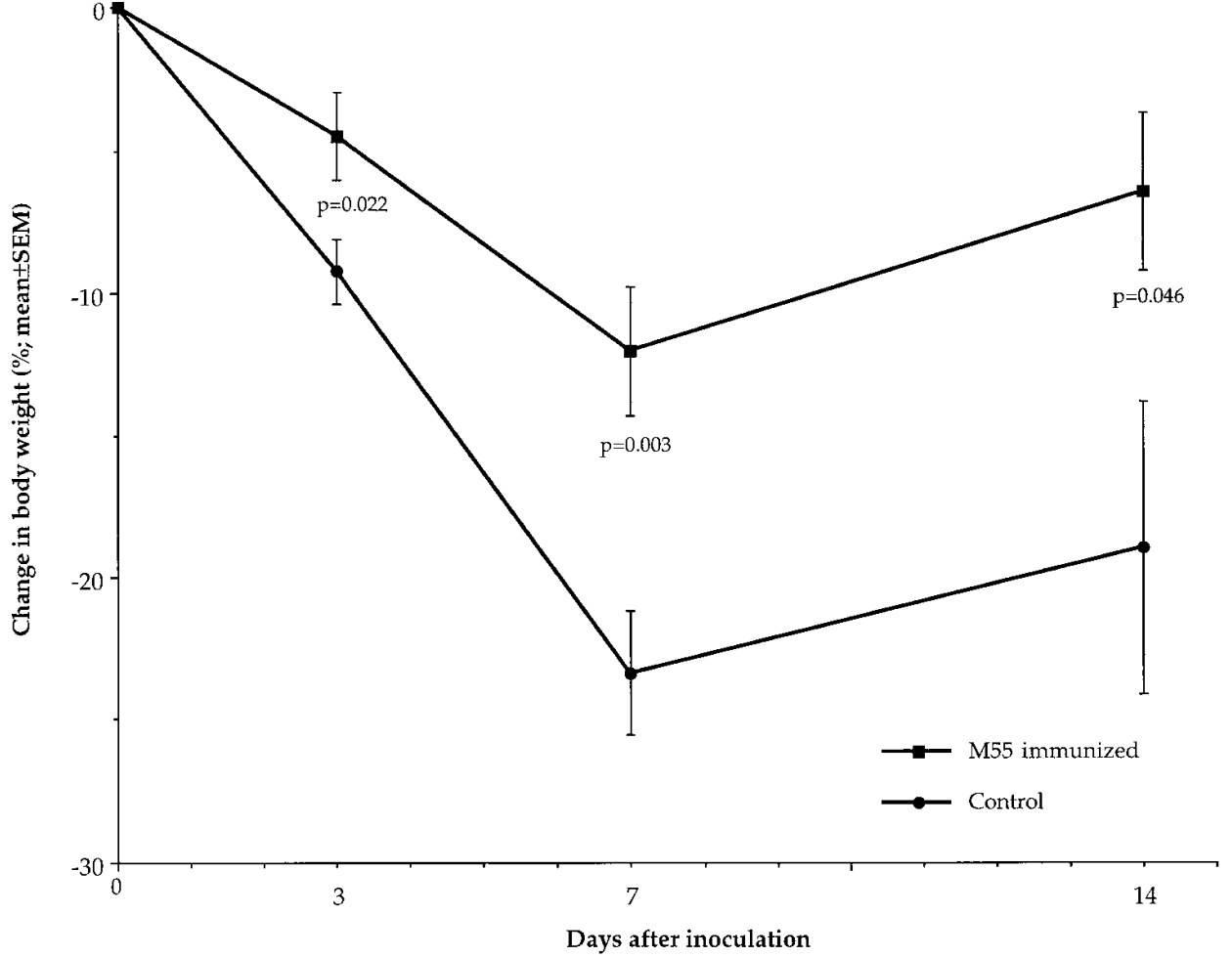

Figure 3. Changes of body weight of NMRI mice immunized with recombinant collagen adhesin M55 $(n=$ $16)$ and BSA $(n=15)$ before i.v. inoculation with $6 \times 10^{6} \mathrm{CFU}$ per mouse $S$. aureus strain Phillips. geted against the cell surface localized collagen adhesin, an additional experiment was conducted. Mice were immunized with M55 $(n=10)$ or BSA $(n=10)$ and challenged i.v. with $2.8 \times 10^{7} \mathrm{CFU}$ per mouse of $S$. aureus LS-1, a strain that does not express the collagen adhesin. In contrast to the protective effect of immunization with M55 seen in the previous three experiments, no statistically significant differences between M55 immunized mice and control mice were found with respect to mortality rate or weight loss. $14 \mathrm{~d}$ after the bacterial inoculation, $50 \%$ of M55 immunized mice had died compared with $30 \%$ of BSA immunized mice.

Clinical outcome of disease-passive immunization. A passive immunization study was conducted to verify that the protection of mice against $\mathrm{CNA}^{+} S$. aureus challenge is mediated by collagen adhesin specific antibodies. Rats were actively immunized with either M55 or BSA and subsequently bled, the sera collected, and pooled. The immunoglobulin fraction from the pooled hyperimmune sera was administered to naive mice by i.p. injection. $24 \mathrm{~h}$ later the passively immunized mice were challenged i.v. with $4 \times 10^{7} \mathrm{CFU}$ per mouse $S$. aureus strain Phillips. One week after bacterial inoculation, only 1 of 10 animals in the anti-M55 immunoglobulin treated group had died, compared with 8 of 10 mice in the control group $(P<0.01)$. The data taken together with the previous results clearly indicate that anticollagen adhesin antibodies, either elicited by active immunization or adoptively transferred, can protect mice against a lethal $S$. aureus challenge.

In vivo immune responses. Murine B lymphocyte responses were evaluated to analyze the protective mechanism provided by antisera resulting from collagen adhesin immunizations. 96well ELISA plates, coated with either $S$ aureus strain Phillips $\left(\mathrm{CNA}^{+}\right)$or $S$. aureus strain LS-1 (CNA $\left.{ }^{-}\right)$, were used to evaluate the humoral response induced in M55 vaccinated mice. As shown in Fig. 4, a strong antibody reaction to $S$. aureus Phillips but not to $S$. aureus LS-1 was seen in the sera obtained from mice immunized with M55. In contrast, sera from mice experimentally infected with $S$. aureus Phillips, but not immunized with M55, recognized molecules expressed by both staphylococcal strains. Normal mouse sera failed to react significantly to either of the $S$. aureus strains. To compare the antibody response induced in mice by immunization with M55 versus experimentally induced infection by $S$. aureus Phillips, microtiter wells were coated with purified recombinant proteins M17, M31, M55, as well as the B1 subunit from the collagen adhesin. Ovalbumin was used as the control antigen. Sera from mice immunized with M55 displayed a strong antibody response against the immobilized collagen adhesin fragments M17, M31, and the M55, but not to B1 subunit or ovalbumin (Fig. 5, $A$ and $B$ ). Increase of the serum M55 IgG antibody levels was even more impressive in mice vaccinated with M55 and then infected when compared with antibody levels in animals vaccinated but not challenged (Fig. $5 A$ ). Notably, there was a significant correlation $\left(r_{\mathrm{s}}=0.46 ; P=0.01\right)$ between anti-M55 antibody levels and the mortality in all mice participating in the experiment. Interestingly, sera from mice immunized with BSA and infected with $S$. aureus Phillips did not elicit antibodies that reacted with any of the purified recombinant collagen adhesin fragments (Fig. 5, $A$ and $B$ ). This lack of reactivity is somewhat surprising since we were able to detect the collagen adhesin on the surface of $S$. aureus strain Phillips, immunohistochemically using antibodies to M55 (results not shown). Moreover, Gillaspy et al. (24) have demonstrated recently in vivo expression of the $S$. aureus collagen adhesin. Mice immunized with M31 displayed a similar magnitude of antibody response when compared with M55 vaccinated animals (2.33 0.09 for M31 $[n=14] ; 1.82 \pm 0.06$ for M55 [ $n=14]$; $1.74 \pm 0.06$ 


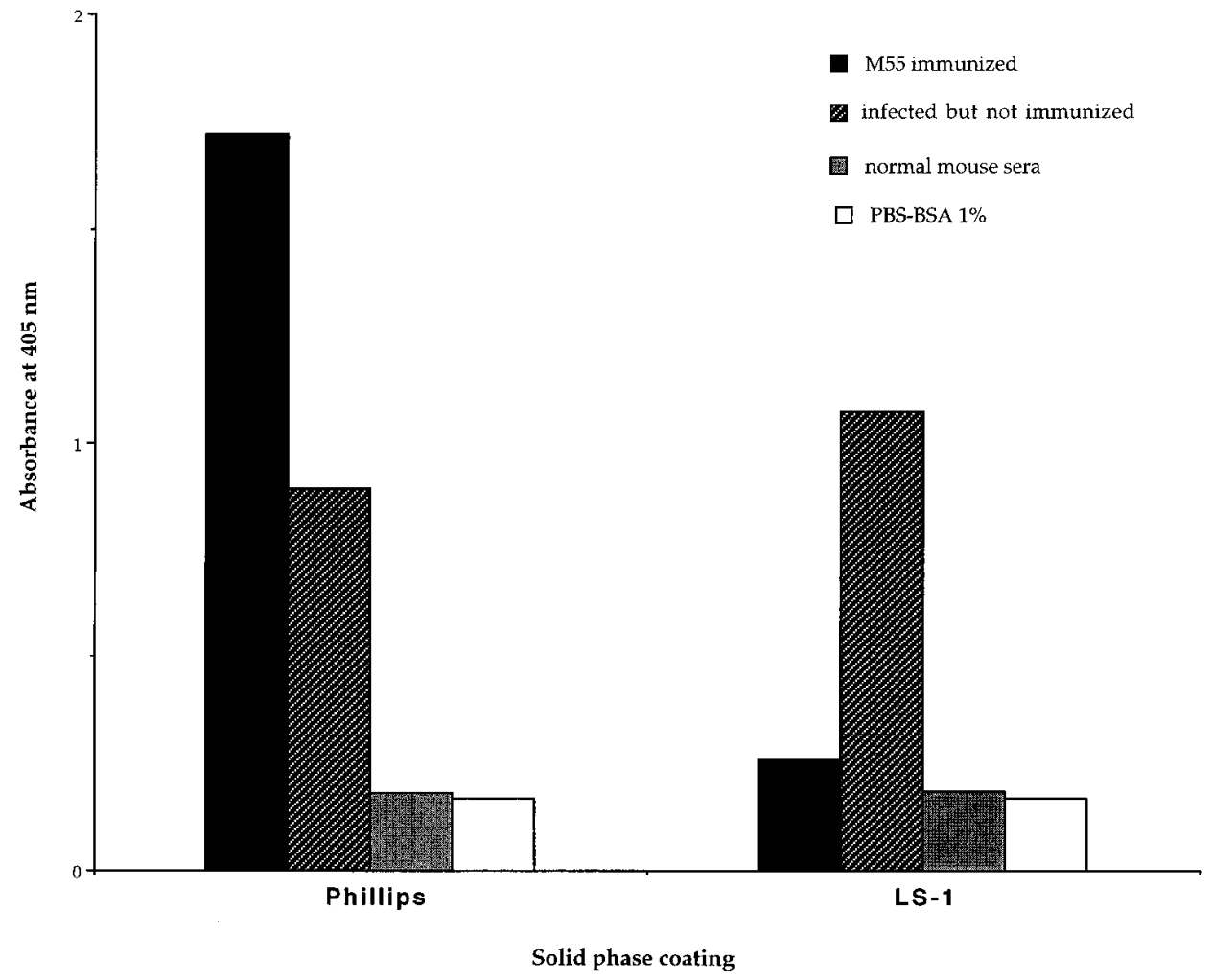

Figure 4. Serum antibody responses to intact bacteria $S$. aureus strain Phillips $\left(\mathrm{CNA}^{+}\right)$and $S$. aureus strain LS-1 $\left(\mathrm{CNA}^{-}\right)$. The mice were immunized with M55, and BSA, or not immunized but experimentally infected i.v. with $S$. aureus strain Phillips. for M17 [ $n=14]$; $0.03 \pm 0.00$ for B1 $[n=14]$; and $0.01 \pm 0.00$ for OVA $[n=14]) .14 \mathrm{~d}$ after inoculation with $S$. aureus Phillips there were no significant differences with respect to serum IL-6 levels between mice immunized with M55 or BSA $(1,768 \pm 258$ versus $2,225 \pm 641$, NS). Splenomegaly, typically observed as a consequence of severe $S$. aureus infection, did not differ between the study groups (data not shown).

Efficient intracellular killing of staphylococci opsonized with collagen adhesin antibodies. Opsonophagocytosis plays a significant role in the clearance of $S$. aureus from infected hosts (25). A series of in vitro and in vivo studies were conducted to evaluate the effect of antibodies to the collagen adhesin on the growth, phagocytosis, and intracellular killing of opsonized $S$. aureus Phillips. To determine the impact of specific anti-M55 antibodies on persistence of bacteria in parenchymatous organs, $S$. aureus strain Phillips $\left(6 \times 10^{6} \mathrm{CFU}\right.$ per
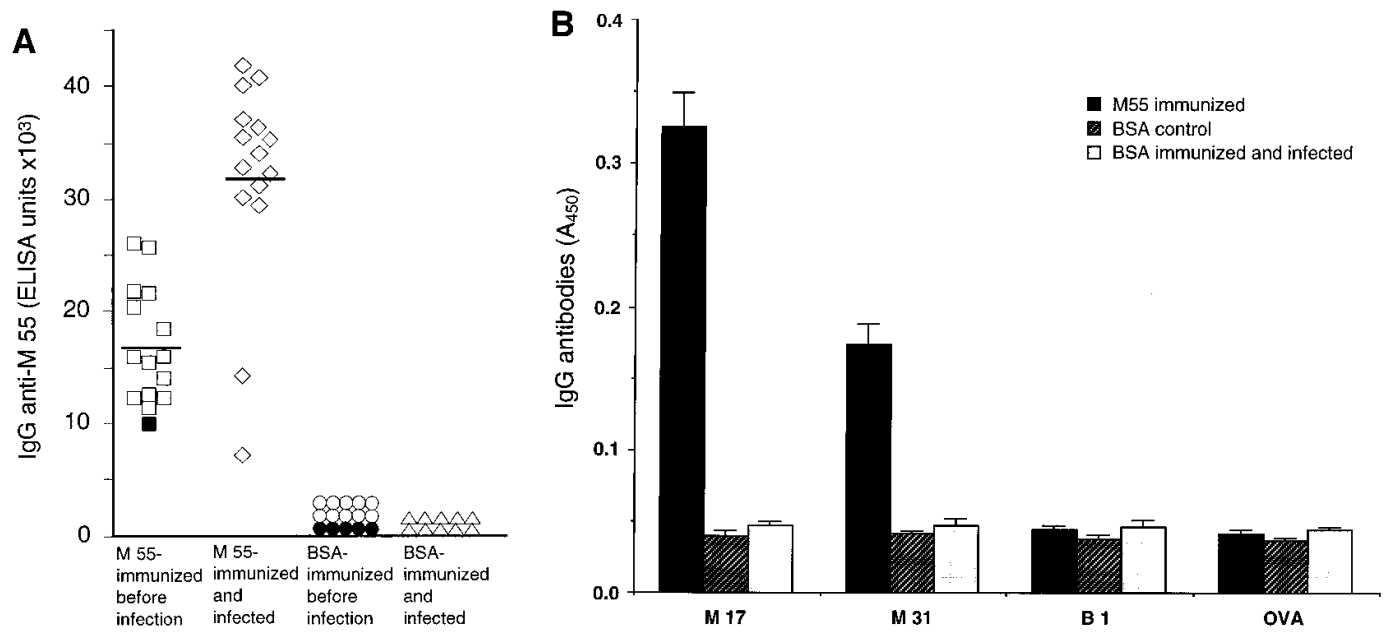

Figure 5. (A) Serum IgG antibody levels (expressed as ELISA units) specific for the M55 fragment of the collagen adhesin. The sera were diluted 1:20,000 and the values represent individual ELISA units. Sera were obtained from mice immunized with M55 $(n=15)$ or BSA $(n=15)$. Both groups of mice were subsequently inoculated with collagen adhesin expressing $S$. aureus strain Phillips, bled $14 \mathrm{~d}$ later, and the sera were assayed for presence of M55 spe-

cific IgG. The black symbols represent mice that succumbed as a result of infection. Horizontal bars represent mean anti-M55 levels. (B) Specific serum IgG antibody levels (mean \pm SEM) against different fragments (M31, M17, and B1 subunit) of the collagen adhesin. Mice were immunized with M55 $(n=15)$ or BSA $(n=15)$ and assayed for the antibody levels before $S$. aureus inoculation. The BSA immunized group was bled another time and assayed for collagen adhesin reactivity 14 days after $S$. aureus inoculation $(n=10)$. OVA was used as a control antigen. All the serum samples were diluted 1:5,000. 

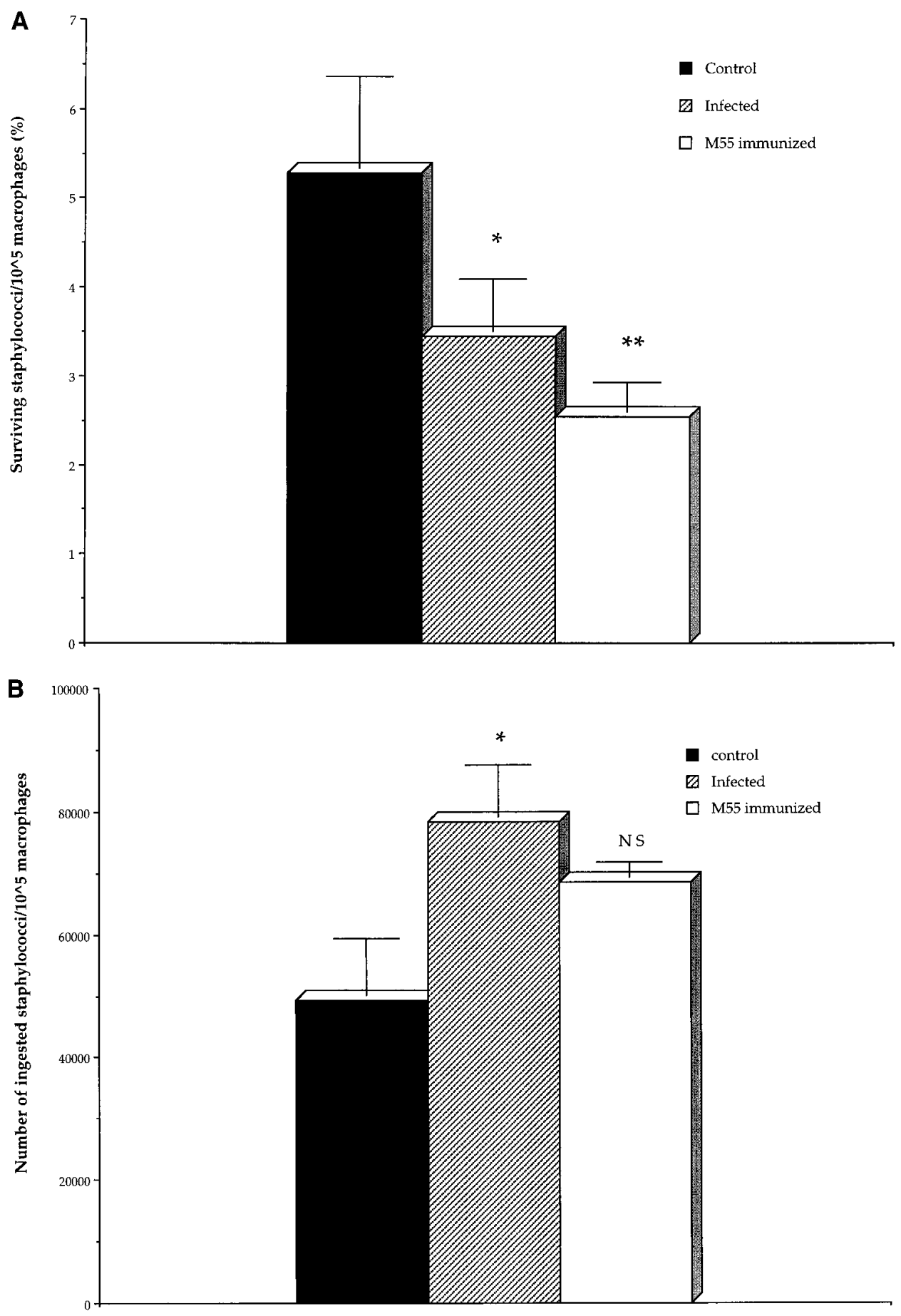

Figure 6. (A) Intracellular killing of bacteria by peritoneal macrophages, obtained from naive mice, after opsonization with heat-inactivated hyperimmune M55 sera-sera from unimmunized mice that have gone through infection with $S$. aureus strain Phillips or BSA hyperimmune sera, respectively. Briefly, macrophages were collected from the peritoneal cavity of NMRI mice, adjusted to $2 \times 10^{6}$ cells $/ \mathrm{ml}$ and seeded into 24-well plates. Opsonized staphylococci were added to the macrophages. After $4 \mathrm{~h}$ of incubation in medium containing minimal inhibitory concentration of gentamicin to avoid extracellular replication of bacteria, the macrophages were lysed with distilled water and the lysate was cultured on agar plates. Values are means \pm SEM. All of the $P$ values provided represent comparisons between sera from control group and from vaccinated or infected groups. $* P<0.05$; ** $P<$ 0.01 . $(B)$ Number of in vitro ingested staphylococci after opsonization with heat-inactivated hyperimmune M55 sera-sera from unimmunized mice that have gone through infection or BSA hyperimmune sera, respectively. Briefly, macrophages from naive mice were collected from the peritoneal cavity, adjusted to $2 \times 10^{6}$ cells $/ \mathrm{ml}$ and seeded into 24 -well plates. Opsonized staphylococci were added to the macrophages. After 50 min of incubation, the macrophages were washed to remove noningested bacteria. To determine the uptake of the opsonized $S$. aureus, macrophages were immediately analyzed. Values are means \pm SEM. All of the $P$ values provided represent comparisons between sera from control group and from vaccinated or infected groups. ${ }^{*} P<0.05$.

mouse) was administered i.v. to mice immunized previously with M55 or BSA. $14 \mathrm{~d}$ after i.v. challenge the kidneys and joints of the immunized mice were aseptically harvested and the bacterial load assessed. The kidneys from the M55 immunized mice contained threefold fewer organisms compared with the kidneys isolated from the BSA immunized animals $\left(1.4 \pm 0.6 \times 10^{7} \mathrm{CFU}\right.$ versus $\left.4.4 \pm 1.9 \times 10^{7} \mathrm{CFU}\right)$. Positive cultures were obtained from the joints in $13 \%$ of M55 immunized mice, compared with $30 \%$ of the joints from the BSA immunized animals. These data suggest that protection provided by immunization with M55 may be mediated by a more efficient clearance of the invading staphylococci. Alternatively, decreased bacterial adherence to collagenous structures (cartilage in case of septic arthritis or subendothelial space in case of sepsis) as well as decreased bacterial proliferation after the initial adherence event cannot be excluded.

To further define the observed decreased bacterial persistence in vivo, we examined the impact of specific antibodies to the collagen adhesin on phagocytosis and intracellular killing capacity of peritoneal macrophages. S. aureus strain Phillips was opsonized with serum from mice immunized with M55, serum from mice immunized with BSA, or alternatively, serum 
from naive mice that had survived a systemic infection with $S$. aureus Phillips. Peritoneal macrophages exposed to $S$. aureus Phillips, opsonized with serum from M55 immunized mice, displayed a significantly enhanced intracellular killing capacity compared with macrophages exposed to control opsonized staphylococci $(P=0.009$; Fig. $6 A)$. The intracellular killing of $S$. aureus strain Phillips by macrophages was also moderately enhanced by pre-opsonization with serum derived from animals previously infected with the homologous strain $(P=0.037)$. In addition to enhanced intracellular killing, the phagocytic capacity of the macrophages was moderately increased by opsonization of $S$. aureus with serum from M55 immunized mice and serum from mice inoculated with strain Phillips (Fig. $6 \mathrm{~B}$ ).

\section{Discussion}

Gram-positive bacterial sepsis is on the increase. In fact, between one-third and one-half of all cases of sepsis are caused by gram-positive bacteria, particularly $S$. aureus and Staphylococcus epidermidis. In the United States, it is estimated that over 200,000 patients will develop gram-positive sepsis this year (26). Patients at risk for $S$. aureus infection include those with impaired host defense mechanisms caused by immunosuppressive drugs, trauma, severe burn, and drug abuse. The emergence of antibiotic resistance among $S$. aureus has made treatment of staphylococcal infections difficult and a vaccine to prevent staphylococcal infections especially for patients at high risk is a great priority. Immunity to $S$. aureus infections remains poorly understood and much remains to be learned about the relative importance of various staphylococcal surface antigens and secreted products in the induction of a protective immunity to $S$. aureus. In general, protection against $S$. aureus infection is attributed to intact epithelial and mucosal barriers and normal cellular and humoral responses.

Over the past several decades live, heat-killed, and formalin fixed preparations of $S$. aureus cells have been tested as vaccines to prevent staphylococcal infections (25). A multicentre clinical trial was designed to study the effects of a commercial vaccine, consisting of a staphylococcus toxoid and whole killed staphylococci, on the incidence of peritonitis, exit site infection, and $S$. aureus nasal carriage among continuous peritoneal dialysis patients (27). Similarly, immunization of rabbits with whole cells of $S$. aureus could not prevent or modify any stage in the development of experimental endocarditis, reduce the incidence of renal abscess, or lower the bacterial load in infected kidneys (28). The lack of protection in these vaccination experiments can be explained by arguments ranging from improper immunization scheduling and suboptimal immunization strategies to failing to generate an adequate immune response.

Immunotherapies designed to inhibit the adhesion of pathogenic microorganisms appear to be an effective way to prevent or minimize the severity of an infection. An increasing understanding of the interactions between adhesins and host components, at the molecular level, has now provided a strong foundation for a new generation of subunit vaccines. In addition to the data provided in this study other vaccine strategies targeting bacterial adhesins are emerging. For example, immunization with FimH, an $E$. coli type 1 pili adhesin, reduced in vivo colonization of bladder mucosa by $>99 \%$ in a murine cystitis model (29). It has also been demonstrated that immu- nization with adhesins can elicit protection against microbial challenge, such as Haemophilus influenzae-induced otitis media in a chinchilla model (30). In most cases, immunization with adhesins leads to the production of specific antibodies that prevent infection by inhibiting bacterial attachment and colonization, as well as enhancing bacterial opsonophagocytosis and antibody-dependent complement-mediated killing.

The collagen adhesin mediates the attachment of $S$. aureus to cartilage (23) and is clearly a virulence determinant in septic arthritis and endocarditis $(12,13)$. In this study, we wanted to evaluate the protective efficacy of immunization with recombinant fragments of the $S$. aureus collagen adhesin. Using a mouse model of $S$. aureus infection (18), we have clearly demonstrated that both active and passive immunization with antibodies directed against the A domain (M55) of the collagen adhesin can protect mice against $S$. aureus sepsis-induced death. Mice were immunized subcutaneously with either M55 or the control antigen, BSA and then challenged i.v. with a heterologous $\mathrm{CNA}^{+} S$. aureus isolate strain Phillips. In three different experiments $83 \%$ (35/42) of the mice actively immunized with M55 survived the bacterial challenges compared with only $27 \%(12 / 45)$ of the mice from the control groups. Moreover, passive immunization of naive mice with hyperimmune sera from M55 immunized rats also provided protection against i.v. S. aureus challenge.

The exact mechanism of protection against $S$. aureus infection induced by immunization with the collagen adhesin is unclear. Early during staphylococcal bacteremia there is a profound systemic release of proinflammatory cytokines (31). This release leads to direct toxic effects on endothelial cells and enhances permeability (32-34). Leakage in vasculature is a prerequisite for extravasation of bacteria from the blood stream to underlying tissues. This extravasation might be enhanced by interactions between the staphylococcal collagen adhesin and subendothelial collagen type IV situated in the blood vessel extracellular matrix. Studies by Vercelotti et al. (35) and House-Pompeo et al. (36) indicate that $S$. aureus specifically interacts with type IV collagen (35). Anticollagen adhesin antibodies may prevent the binding of staphylococci to subendothelial collagen type IV, thereby giving rise to a more favorable clinical outcome. It is interesting to note that immunization with M55 provided better protection against i.v. S. aureus challenge compared with the smaller constructs M17 and M31. Previously, we have shown that the recombinant protein M55 exhibits a 100-fold higher affinity to collagen compared with the smaller constructs (36). In addition, monoclonal antibodies raised against M55, but mapped to epitopes outside M17 and M31, can inhibit S. aureus binding to collagen (unpublished results). Therefore, it is possible that the antibodies raised against M55 are more effective in preventing extravasation of $S$. aureus from the blood stream to the subendothelial type IV collagen. It has been demonstrated that fewer bacteria were recovered from the kidneys and joints of mice actively immunized with M55 compared with organs from BSA immunized animals. In addition, a significant enhancement of the intracellular killing capacity of peritoneal macrophages was demonstrated against $S$. aureus opsonized with M55 hyperimmune sera compared with $S$. aureus opsonized with control sera or sera from mice that had been experimentally infected.

Infection with $\mathrm{CNA}^{+} S$. aureus strain Phillips did not induce a detectable immune response to collagen adhesin, at least as judged by antibody levels measured in the ELISA. As 
evidenced in our study, this is not due to a lack of in vivo expression of this molecule on the bacterial cell surface. The inability to generate a sufficient antibody response to the collagen adhesin results in an ineffective clearance of the invading bacteria. Indeed, a significant correlation was noted between serum levels of anti-M55 antibodies and mortality $\left(r_{\mathrm{s}}=0.46 ; P=\right.$ $0.01)$ as well as weight decrease, the latter finding being another parameter of severity of infection $\left(r_{\mathrm{s}}=0.49 ; P=0.009\right.$ at day 7 after $S$. aureus inoculation and $r_{\mathrm{s}}=0.40 ; P=0.056$ at day 14 after S. aureus inoculation). Furthermore, the only mouse in the M55 immunized group that died during the course of the experiment displayed low levels of M55 specific antibodies (Fig. 5 A). Notably, immunogenecity of the collagen adhesin molecule itself is not the problem, since immunization with the recombinant protein induced high levels of specific and protective antibodies. The exact reason why collagen adhesin is not recognized by the immune system remains a matter of intense study. It is quite possible that induction of polyclonal B cell activation, as a consequence of infection with whole $S$. aureus organisms, may downregulate rather than upregulate specific immune responsiveness to a variety of bacterial cell wall components. Perhaps such an inability to raise protective immunological memory in response to exposure to $S$. aureus could explain the recurrent infection pattern of staphylococcal diseases.

Using an experimental model of sepsis initiated by i.v. injection of $S$. aureus, we have demonstrated that vaccination with a recombinant version of the collagen adhesin protect mice against a heterologous challenge of $\mathrm{CNA}^{+} S$. aureus. Immunization with the collagen adhesin profoundly altered the course of disease by decreasing the mortality rate up to sixfold when compared with control mice. Since expression of collagen adhesin is common among invasive $S$. aureus isolates (37), this adhesin should be included in the development of future $S$. aureus vaccines.

\section{Acknowledgments}

We thank Lena Svensson, Margareta Verdrengh, Helena Kahu, and Amy Schneider for excellent technical assistance.

This work was supported by grants from Göteborg Medical Society, the Swedish Association against Rheumatism, the King Gustaf V's 80 Years Foundation, the Swedish Medical Research Council, the Nanna Svartz Foundation, the A.-G. Crafoord Foundation, the University of Göteborg, Börje Dahlin Foundation, the National Institutes of Health (AR44415), the Arthritis Foundation, and Inhibitex, Inc.

\section{References}

1. Foster, T.J. 1991. Potential for vaccination against infections caused by Staphylococcus aureus. Vaccine. 9:221-227.

2. Patti, J.M., B.L. Allen, M.J. McGavin, and M. Höök. 1994. MSCRAMMmediated adherence of microorganisms to host tissues. Annu. Rev. Microbiol. 48:585-617.

3. Patti, J.M., and M. Höök. 1994. Microbial adhesins recognizing extracellular matrix macromolecules. Curr. Opin. Cell Biol. 6:752-758.

4. Flock, J.I., G. Fröman, K. Jönsson, B. Guss, C. Signäs, B. Nilsson, G. Raucci, M. Höök, T. Wadström, and M. Lindberg. 1987. Cloning and expression of the gene for fibronectin-binding protein from Staphylococcus aureus. EMBO (Eur. Mol. Biol. Organ.) J. 6:2351-2357.

5. McDevitt, D., P. Francois, P. Vaudaux, and T.J. Foster. 1994. Molecular characterization of the clumping factor (fibrinogen receptor) of Staphylococcus aureus. Mol. Microbiol. 11:237-248.

6. Park, P.W., J. Rosenbloom, W.R. Abrams, and R.P. Mecham. 1996. Molecular cloning and expression of the gene for elastin-binding protein (ebpS) in Staphylococcus aureus. J. Biol. Chem. 271:15803-15809.
7. Patti, J.M., H. Jonsson, B. Guss, L.M. Switalski, K. Wiberg, M. Lindberg, and M. Höök. 1992. Molecular characterization and expression of a gene encoding a Staphylococcus aureus collagen adhesin. J. Biol. Chem. 267:4766-4772.

8. Vaudaux, P.E., D.P. Lew, and F.A. Waldvogel. 1989. Host factors predisposing to and influencing therapy of foreign body infections. In Infections Associated with Indwelling Medical Devices. A.L. Bisno and F.A. Waldvogel, editors. American Society for Microbiology, Washington, DC. 1-29.

9. Kochwa, S., R.S. Litwak, R.E. Rosenfield, and E.F. Leonard. 1977. Blood elements at foreign surfaces: a biochemical approach to study the adsorption of plasma proteins. Ann. NY Acad. Sci. 283:37-49.

10. Vaudaux, P., D. Pittet, A. Haeberli, E. Huggler, U.E. Nydegger, D.P. Lew, and F.A. Waldvogel. 1989. Host factors selectively increase staphylococcal adherence on inserted catheters-a role for fibronectin and fibrinogen or fibrin. J. Infect. Dis. 160:865-875.

11. Vaudaux, P., D. Pittet, A. Haeberli, P.G. Lerch, J.J. Morgenthaler, R.A Proctor, F.A. Waldvogel, and D.P. Lew. 1993. Fibronectin is more active than fibrin or fibrinogen in promoting Staphylococcus aureus adherence to inserted intravascular catheters. J. Infect. Dis. 167:633-641.

12. Patti, J.M., T. Bremell, D. Krajewska-Pietrasik, A. Abdelnour, A. Tarkowski, C. Rydén, and M. Höök. 1994. The Staphylococcus aureus collagen adhesin is a virulence determinant in experimental septic arthritis. Infect. Immun. 62:152-161.

13. Heinz, S.A., T. Schennings, A. Heimdahl, and J.-I. Flock. 1996. Collagen binding of Staphylococcus aureus is a virulence factor in experimental endocarditis. J. Infect. Dis. 174:83-88.

14. Bremell, T., S. Lange, L. Svensson, E. Jennische, K. Gröndahl, H. Carlsten, and A. Tarkowski. 1990. Outbreak of spontaneous staphylococcal arthritis and osteitis in mice. Arthritis Rheum. 33:1739-1744.

15. Patti, J.M., K. House-Pompeo, J.O. Boles, N. Garza, S. Gurusiddappa, and M. Höök. 1995. Critical residues in the ligand-binding site of the Staphylococcus aureus collagen-binding adhesin (MSCRAMM). J. Biol. Chem. 270: 12005-12011.

16. Patti, J.M., J.O. Boles, and M. Höök. 1993. Identification and biochemical characterization of ligand binding domain of the collagen adhesin from Staphylococcus aureus. Biochemistry. 32:11428-11435.

17. Mancini, G., A.O. Carbonara, and J.F. Heremans. 1965. Immunochemical quantitation of antigens by single radial immunodiffusion. Immunochemistry. 2:235-254.

18. Bremell, T., S. Lange, A. Yacoub, C. Rydén, and A. Tarkowski. 1991. Experimental Staphylococcus aureus arthritis in mice. Infect. Immun. 59:26152623.

19. Bremell, T., A. Abdelnour, and A. Tarkowski. 1992. Histopathological and serological progression of experimental Staphylococcus aureus arthritis. Infect. Immun. 60:2976-2985.

20. Helle, M., L. Boeije, and L. Aarden. 1988. Functional discrimination between interleukin 6 and interleukin 1. Eur. J. Immunol. 18:1525-1540.

21. Lissner, C.R., R. Swanson, and A.D. O'Brian. 1983. Genetic control of the innate resistance of mice to Salmonella typhimurium: expression of the Ity gene in peritoneal and splenic macrophages isolated in vivo. J. Immunol. 131: 3006-3013.

22. Schneewind, O., D. Mihaylovapetkov, and P. Model. 1993. Cell wall sorting signals in surface proteins of gram-positive bacteria. EMBO (Eur. Mol. Biol. Organ.) J. 12:4803-4811.

23. Switalski, L.M., J.M. Patti, W. Butcher, A.G. Gristina, P. Speziale, and M. Höök. 1993. A collagen receptor on Staphylococcus aureus strain isolated from patients with septic arthritis mediates adhesion to cartilage. Mol. Microbiol. 7:99-107.

24. Gillaspy, A.F., J.M. Patti, and M.S. Smeltzer. 1997. Transcriptional regulation of the Staphylococcus aureus collagen adhesin gene, cna. Infect. Immun. 65:1536-1540

25. Lee, J.C. 1996. The prospects for developing a vaccine against Staphylococcus aureus. Trends Microbiol. 4:162-166.

26. Bone, R.C. 1994. Gram-positive organisms and sepsis. Arch. Intern. Med. 154:26-34.

27. Poole-Warren, L.A., M.D. Hallett, P.W. Hone, S.H. Burden, and P.C. Farrell. 1991. Vaccination for prevention of CAPD associated staphylococcal infection: results of a prospective multicentre clinical trial. Clin. Nephrol. 35: 198-206.

28. Greenberg, D.P., I.J. Ward, and A.S. Bayer. 1987. Influence of Staphylococcus aureus antibody on experimental endocarditis in rabbit. Infect. Immun. 55:3030-3034.

29. Langermann, S., S. Palaszynski, M. Barnhart, G. Auguste, J.S. Pinker, J. Burlein, P. Barren, S. Koening, S. Leath, C.H. Jones, and S.J. Hultgren. 1997. Prevention of mucosal Escherichia coli infection by FimH-adhesin-based systemic vaccination. Science. 276:607-611.

30. Sirakowa, T., P.E. Kolattukudy, D. Murwin, J. Billy, E. Leake, D. Lim, T. Demaria, and L. Bakaletz. 1994. Role of fimbriae expressed by nontypeable Haemophilus influenzae in pathogenicis of an protection against otitis media and relatedness of the fimbrine subunit to outer membrane protein A. Infect. Immun. 62:2002-2020.

31. Dinarello, C.A. 1996. Cytokines as mediators in pathogeneis of septic shock. In Pathology of Septic Shock. Current Topics in Microbiology and Im- 
munology, Vol. 216. E.T. Rietschel and H. Wagner, editors. Springer-Verlag, Berlin. 133-165.

32. Martin, C., P. Saux, J.L. Mege, G. Perrin, L. Pepazin, and F. Gouin. 1994. Prognostic values of serum cytokines in septic shock. Intensive Care Med. 20:272-277.

33. Robaye, B., R. Mosselmans, W. Fiers, J.E. Dumont, and P. Galand. 1991. Tumor necrosis factor induces apoptosis (programmed cell death) in normal endothelial cells in vitro. Am. J. Pathol. 138:447-453.

34. Schuger, L., J. Varani, R.M. Marks, S.L. Kunkel, K.J. Johnson, and P.A. Ward. 1989. Cytotoxity of tumor necrosis factor-alpha for human umbilical vein endothelial cells. Lab. Invest. 61:62-68.

35. Vercellotti, G.M., J.B. McCarthy, P. Lindholm, P.K. Peterson, H.S. Jacob, and L.T. Furcht. 1985. Extracellular matrix proteins (fibronectin, Laminin, and type IV collagen) bind and aggregate bacteria. Am. J. Pathol. 120:13-21.

36. House-Pompeo, K., J.O. Boles, and M. Höök. 1994. Characterization of bacterial adhesin interactions with extracellular matrix components utilizing biosensor technology. Methods: A Companion to Methods Enzymol. 6:134-142.

37. Holderbaum, D., T. Spech, L.A. Ehrhart, T. Keys, and G.S. Hall. 1987. Collagen binding in clinical isolates of Staphylococcus aureus. J. Clin. Microbiol. 25:2258-2261. 